\title{
Spatial correlation in 2D and 3D thin films of conserved binary mixtures in the presence of wetting of substrates by the preferred majority component: interpretation in real scenario
}

\author{
Satya Pal Singh
}

Received: 29 November 2011/ Accepted: 14 March 2012/Published online: 15 May 2012

(C) The Author(s) 2012. This article is published with open access at Springerlink.com

\begin{abstract}
The spinodal decomposition has got fresh attraction in the past few decades with the advent of new computational problems under very thin geometries. The nanodrops evolve in the phase separation process. The phase separation process itself interplays with the wetting forces and give rise to structures of importance for a wide range of technological applications from spherical nanomagnetic domains to magnetic strips. MC simulation programs for 2D and 3D cases have been written for surface directed phase separation (using metropolis algorithm) to observe the spatial correlation varying with time, which shows a polynomial fitting behavior for 2D case and follow a peculiar trend with time specially, in early stages of evolution indicating a colossal behavior. The two point correlations (Pearson's linear $x y$ correlation function) if evaluated in $3 \mathrm{D}$ case do not show any important oscillatory behavior but instead confirm for the two regimes as phase separation or mixing and the wetting ones. The correct generalization of $x y$ correlation as $x y z$ correlation in $3 \mathrm{D}$ case (i.e., product of the three moments) does not seem to be a reliable one because it moves to six to seven decimal places, thus comes out at the cost of loss in confidence limit. Thus, the 3D simulation confirm the two regime behavior indicating that the same colossal behavior of $2 \mathrm{D}$ case can exist in real 3D thin film of random binary mixture. Thus, the colossal behavior as obtained for the case of $2 \mathrm{D}$ problem is retained, and this may indicate for a quantized or discrete colossal behavior for certain set of
\end{abstract}

S. P. Singh $(\square)$

Department of Applied Sciences, MMM Engineering College, Gorakhpur 273010, Uttar Pradesh, India

e-mail: singh.satyapal@hotmail.com; spsas@mmmec.in composition and interface parameters for definite but small time periods. The corresponding density profiles are also plotted to confirm the distributions of the two components. Such computational studies may help in developing theoretical models for the observed phenomena and to search out for new events at the very bottom of the scale.

Keywords SDPS · Nanodomains - Spatial correlation · Wetting crossover

\section{Introduction}

Surface directed phase separation (Puri and Binder 1992; Singh et al. 2008; Singh 2011) and wetting phenomena by the preferred component of binary liquid mixtures (Bonn and Ross 2001; Wilding 2003; Binder et al. 1997; Woywod and Schoen 2004; Henderson 1999; Christenson 2001; Singh 2010) have become a problem of extensive study among the scientists in the last 2-3 decades with the advent of fast computing resources. Though first studied experimentally, it could be verified using computer simulations with periodic boundary conditions based on phase transitions achieved in the thermodynamic limit. The new parameters as interface potentials have got introduced to observe the interplay of the mixing or phase separation and the wetting potentials. It has been observed to change the dynamics as well as the structural properties of the domains formed in the process. There seems to be a possible engineering which can yield small drops of the two components of desired shapes and sizes depending on the compositions of the two components, the surface potentials and the interface geometries. The problem of surface directed phase separation can help in exploring the wetting and spinodal dewetting (i.e., rupturing of thin nanofilms of 
thickness $<100 \mathrm{~nm}$ on planar surfaces and in confined geometries) phenomena of thin films (Herminghaus et al. 1998; Sharma and Khanna 1998; Bischof et al. 1996; Reiter 1992, 1998; Xie et al. 1998). Studying the case with conserved compositions with Kawasaki exchange and Glauber kinetic formula can also help in exploring the structural and magnetic properties of binary liquid mixtures with permanent magnetic moments during phase separation under the influence of wetting forces, i.e., preferential attraction for one component, in the case of majority component. The type of simulation and study done in this paper can also reveal the spin wave phenomena and their relaxation in the presence of wetting of boundary walls of domains formed in the process, thus indicating the importance for modeling and development of theory from the molecular interactions point of view.

\section{Methodology and computational procedure}

NVT Monte Carlo simulation programs in 2D and 3D in fortran-77 have been developed with period boundary conditions with a confinement by upper and lower functional walls. A simulation lattice box of size $200 \times 100$ and $100 \times 100 \times 50$ are taken, respectively, in 2D and 3D which represent the quantity $n_{x} \times n_{y}$ and $n_{x} \times n_{y} \times n_{z}$. Computer simulation is done with two component random mixtures of $A$ and $B$, respectively, composed of spin +1 and spin -1 . The majority component spin down $(+1)$ and minority component spin up $(-1)$ were initially placed randomly on the different lattice points in different compositions. The two substrates upper and lower exhibits repulsive interaction with type $\mathrm{B}$ component (spin -1) and preferential attraction for type A component $($ spin +1$)$. In this work, extensive numerical simulations are performed specially for the 3D case to verify the findings reported in Singh (2011) for 2D case. The interactions for which simulations are performed are- $E(W-A)=-1.0,-2.0$, 3.0 and $E(W-B)=1.0,2.0,3.0$ for different compositions as-60:40; 70:30 and 80:20 at temperatures $T=0.60,0.70$ and 0.80 . Glauber Kinetics function [0.5*(1- $\tanh (\Delta E / 2 k B T)]$ (Binder and Heermann 1998) has been chosen to calculate transition probability for achieving detail balance condition. Semi-infinite periodic boundary condition has been employed for the model system, because it is not plausible to work with infinitely large system to get correct picture of the time evolution. Rather, the boundary condition applied in $X$ and $Y$ direction makes it possible to achieve the thermodynamic limit $\mathrm{N} \rightarrow \infty$ (Goldenfeld 1992). Adoption of semi-infinite periodic boundary condition for $X$ and $Y$ direction of lattice means:

$$
\begin{aligned}
& \quad S(0, j, k)=S\left(n_{x}, j, k\right) ; \quad S(0,0, k)=S\left(n_{x}, n_{y}, k\right) ; \\
& \quad S(i, 0, k)=S\left(i, n_{y}, k\right) \\
& S\left(n_{x}+1, j, k\right)=S(1, j, k) ; \quad S\left(n_{x}+1, n_{y}+1, k\right) \\
& \quad=S(1,1, k) ; \quad S\left(i, n_{y}+1, k\right)=S(i, 1, k)
\end{aligned}
$$

Random numbers have been generated using standard ran2 (idum) function program (Press et al. 1998). Kawasaki spin exchange procedure is adopted in which each spin of the lattice is chosen one by one in a regular manner and one out of its six nearest neighbors can be taken corresponding to a random number, thus counting total six possibilities of choosing the nearest neighbor. The free energy has been calculated by exchanging the two spins. If $P(x) \geq \operatorname{rand}(i)$, the exchange of the spins or the move has been accepted where rand $(i)$ is the corresponding random number generated in the process; otherwise, the spins are restored to their original positions. The equality does not allow the system to be trapped in any local minimum of free energy. All the computations have been carried out on a PC with i3 processor.

The Pearson's linear correlations (Press et al. 1998) of $X-Y$ co-ordinates of the two components and their average number densities along $X, Y$ and $Z$ directions are evaluated after every $25 \mathrm{MC}$ cycles. The different time configurations are also stored during the run. All the jobs are run for 28,000 MC cycles.

\section{Results and discussion}

Figure 1 shows the spatial correlation plot of the majority component $\mathrm{A}$ and minority component $\mathrm{B}$ for simulation with a 2D lattice box of size $200 \times 100$. It can be observed that the two curves cross each other in an oscillatory and periodic manner changing the values between positive and

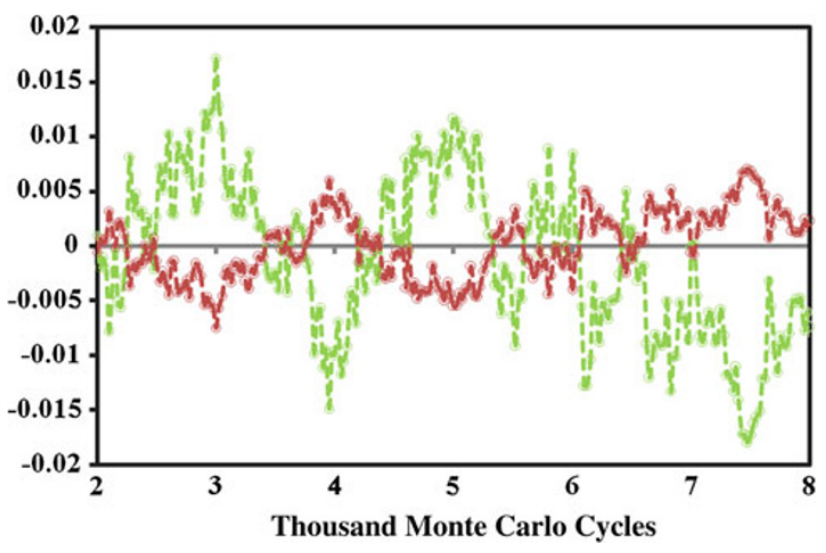

Fig. 1 The spatial correlation plot of the majority component A and minority component $\mathrm{B}$ for simulation with a $2 \mathrm{D}$ lattice box of size $200 \times 100$ 


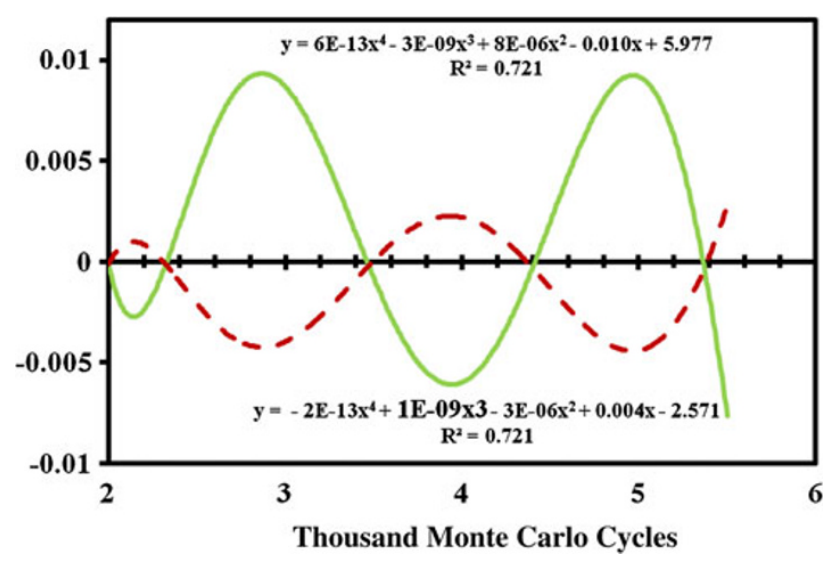

Fig. 2 Correlations for the two components A and B fit very well to higher order polynomials 2D case for early stages of evolution

negative values. The correlations for the two components A and B fit very well to higher order polynomials as shown in Fig. 2, with significant confidence limit but only for early stages of evolution say up to few thousand Monte Carlo cycles. In the latter phases of the evolution, the oscillatory behavior appears but with a difference that it does not fit to the same or similar type of polynomial. This can be explained on the basis of the existence of two prominent regimes. In early stages, the phase separation of the two components takes place interplaying with the wetting surface potential. In the latter stages when wetting of the substrate by the majority component dominate, the inherent non-linearity in the wetting forces diminishes the exact

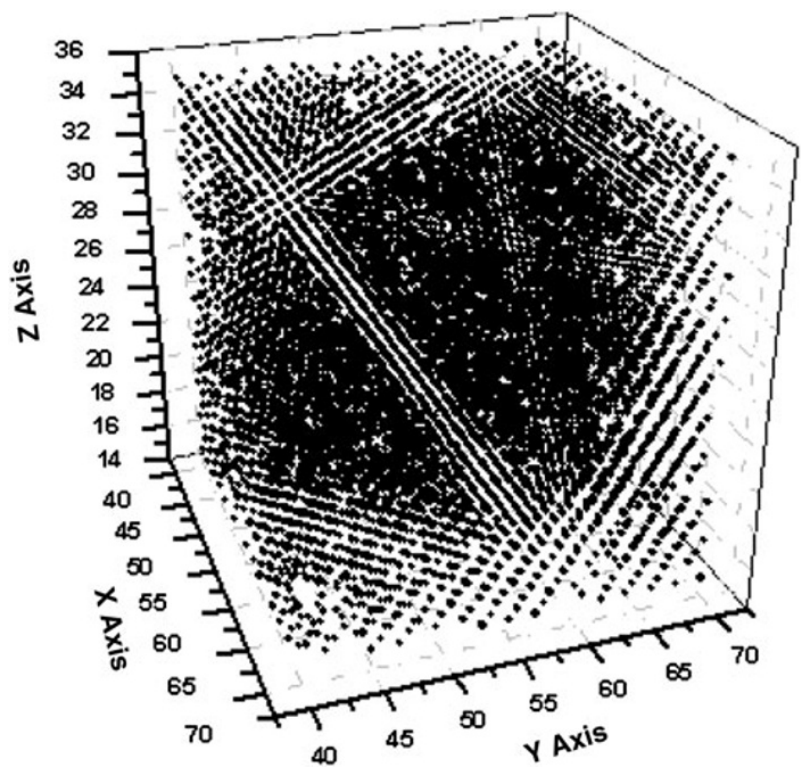

Fig. 3 The coarse graining of the minority component $\mathrm{B}$ in a slab of $40 \times 40 \times 30$ at $T=0.60$ and $\rho_{\mathrm{A}}: \rho_{\mathrm{B}}=0.60: 0.40$ with $E(W-A)=$ +2.0 and $E(W-B)=-2.0$. The darker regions indicate the formation of nanodrops or the precipitates in the case of 3D lattice based NVT-MC simulation polynomial fitting behavior. This type of collective and rhythmic behavior of the lattice points of the simulation cell as a whole do not appear in the case of a 3D thin film. A micrograph of a thin slab in $X$ direction is shown for the majority preferred component. The darker regions indicate the formation of nanodrops or precipitates (Fig. 3). Extensive simulations for the $3 \mathrm{D}$ case with various parameters as surface potential $E(W-A)=-3.0,-2.0$, -1.0 and $E(W-B)=+3.0,+2.0,+1.0$ have been performed for compositions as $\rho_{\mathrm{A}}: \rho_{\mathrm{B}}=0.60: 0.40 ; 0.70: 0.30$ and 0.80:0.20 at temperatures $T=0.60,0.70$ and 0.80 . Figure $4 \mathrm{a}-\mathrm{c}$ show the two point $x y$ correlation versus MC
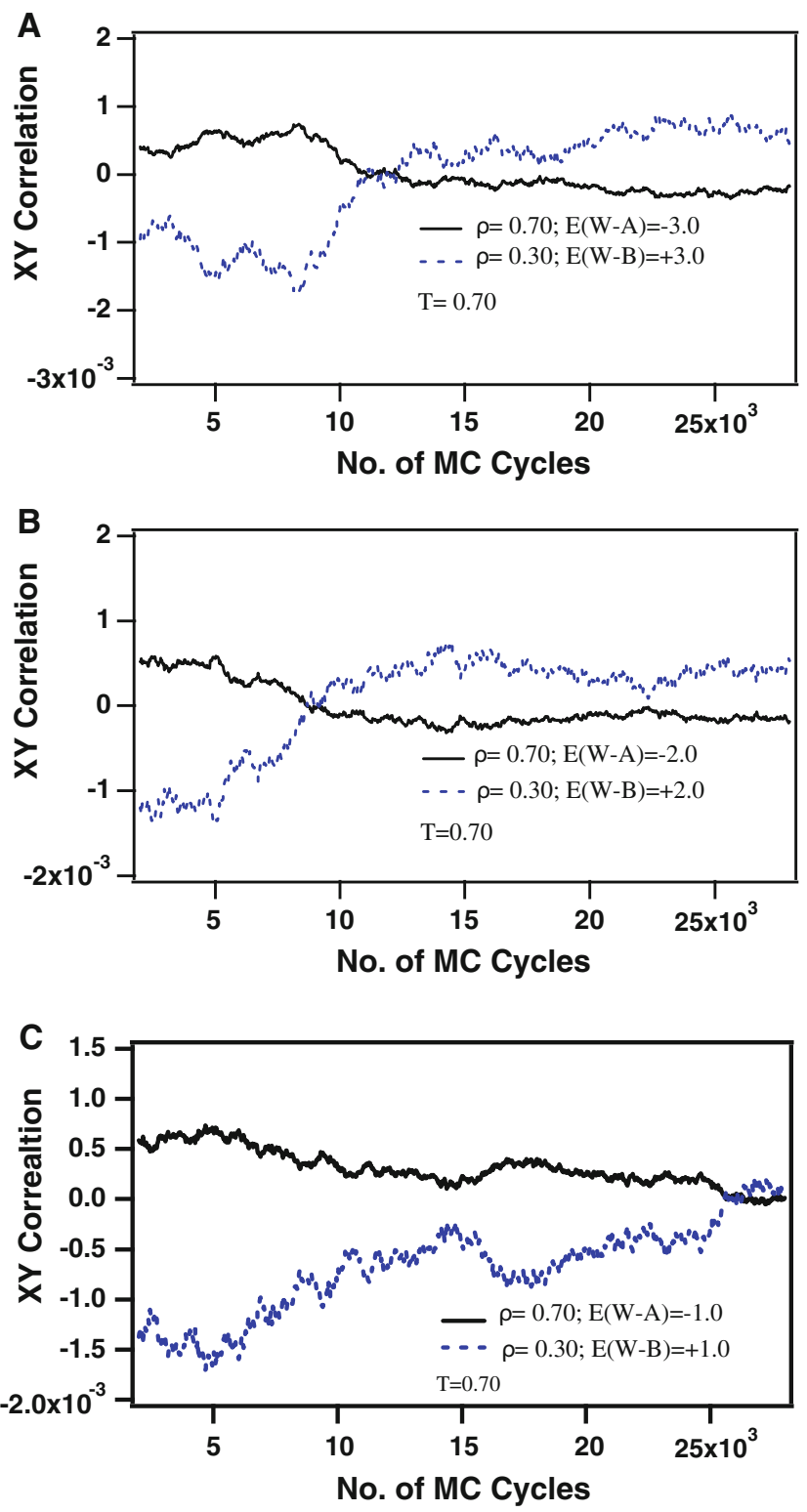

Fig. 4 The two point $x y$ correlation versus $\mathrm{MC}$ cycles for the two components for surface potentials: a $E(W-A)=-3.0$ and $E(W-B)=+3.0 ; \mathbf{b} E(W-A)=-2.0$ and $E(W-B)=+2.0$ at temperature $T=0.70$ with $\rho_{\mathrm{A}}: \rho_{\mathrm{B}}=0.70: 0.30$ 

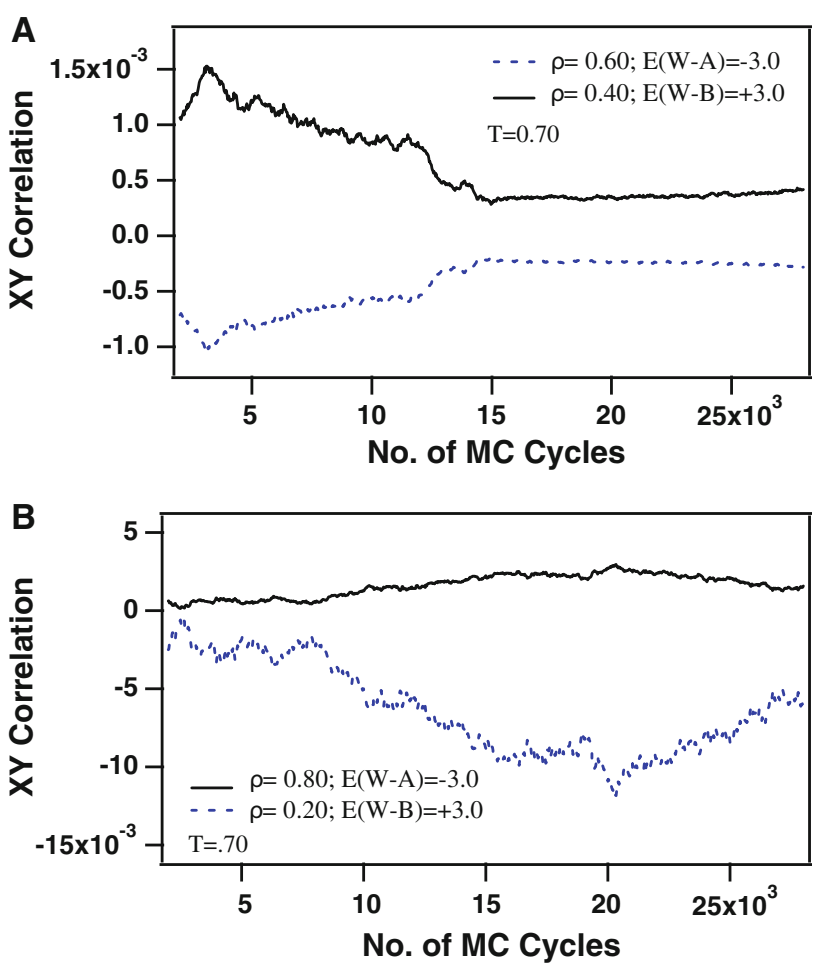

Fig. $5 x y$ correlation for compositions a $\rho_{\mathrm{A}}: \rho_{\mathrm{B}}=0.60: 0.40$ and $\mathbf{b} \rho_{\mathrm{A}}: \rho_{\mathrm{B}}=0.80: 0.40$ at $T=0.70$ and $E(W-A)=-3.0$ and $E(W-B)=+3.0$
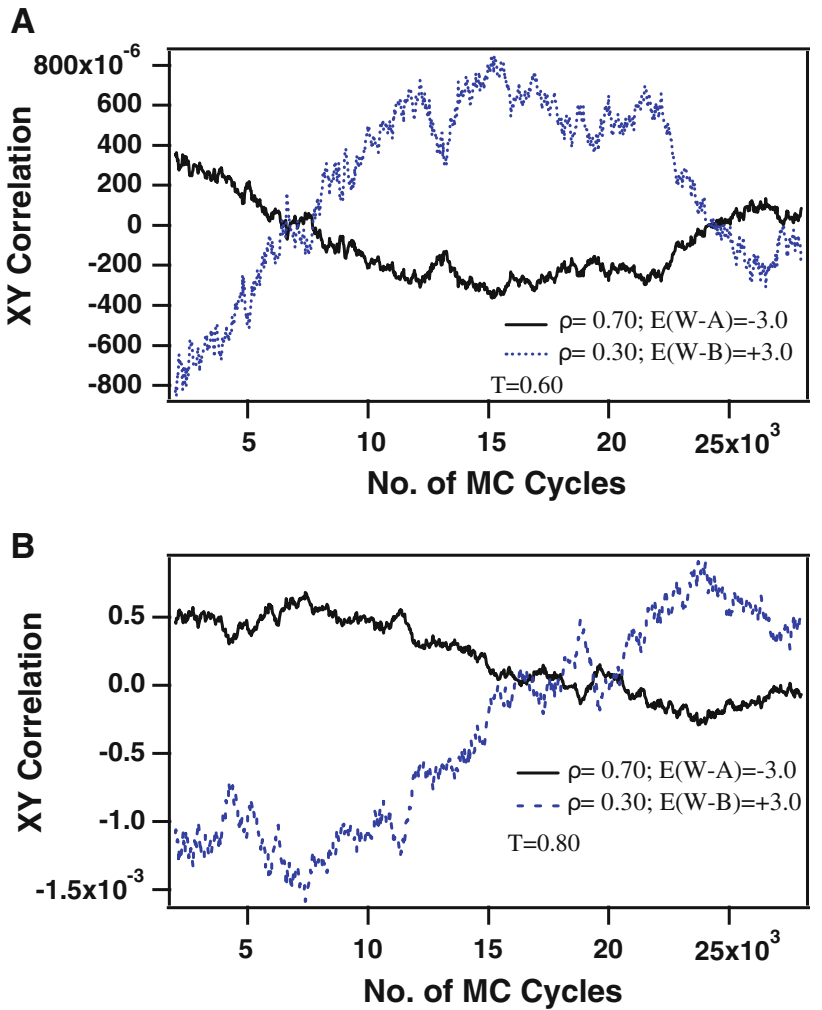

Fig. 6 The correlation at a $T=0.60$ and $\mathbf{b} T=0.80$, respectively, for $\rho_{\mathrm{A}}: \rho_{\mathrm{B}}=0.70: 0.30$

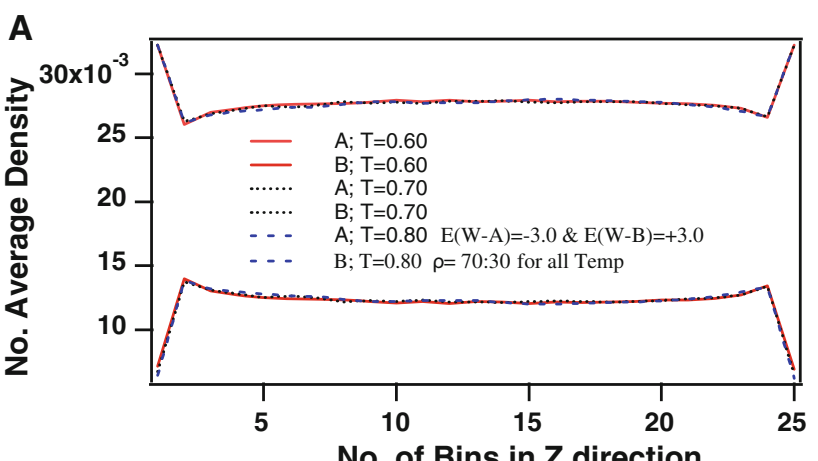

No. of Bins in Z direction

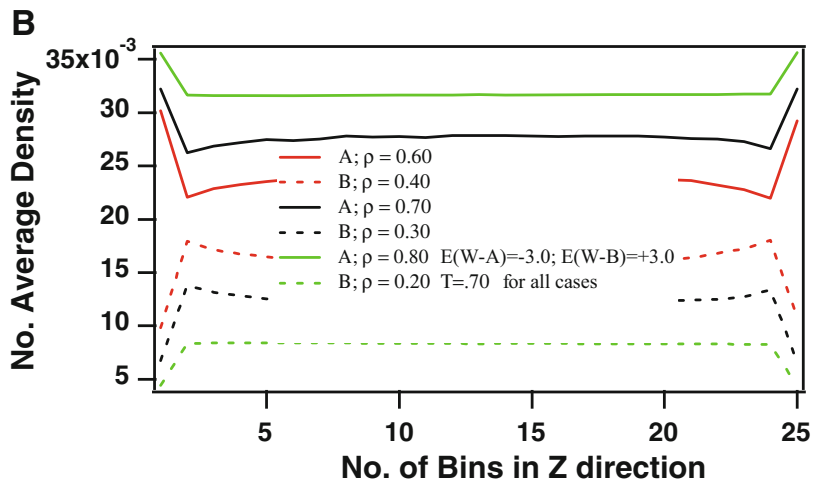

Fig. 7 a The average number density perpendicular to the two walls versus $T$ for $E(W-A)=-3.0$ and $E(W-B)=+3.0$ and $\rho_{\mathrm{A}}: \rho_{\mathrm{B}}=0.70: 0.30$. $\mathbf{b}$ The average number density perpendicular to the two walls versus composition for $E(W-A)=-3.0$ and $E(W-B)=+3.0$ and $T=0.70$

cycles for the two components for surface potentials: $E(W-A)=-3.0,-2.0,-1.0$ and $E(W-B)=+3.0$, $+2.0,+1.0$ at temperature $T=0.70$ with $\rho_{\mathrm{A}}: \rho_{\mathrm{B}}=$ 0.70:0.30. Instead of observing any rhythmic and non-linear response, we observe that the two curves intersect and crossover each other indicating and confirming for the presence of two regime phenomenon. One predominated by phase separation and the other one by the wetting force. For the strong surface potentials, the crossover appears at earlier stage of evolution process. Important thing is to note that this type of crossover is not observed for compositions $\rho_{\mathrm{A}}: \rho_{\mathrm{B}}=0.60: 0.40$ and $\rho_{\mathrm{A}}: \rho_{\mathrm{B}}=0.80: 0.40$ (Fig. 5a, b). This indicates that the two processes do not significantly interplay to affect each other, so that the crossover could not take place. Figure $6 \mathrm{a}, \mathrm{b}$ shows the correlation at $T=0.60$ and $T=0.80$, respectively, for $\rho_{\mathrm{A}}: \rho_{\mathrm{B}}=$ 0.70:0.30. The minimal effect of temperature can be observed in Fig. 7a. The average number density perpendicular to the two walls versus $T$ shows that the wetting of the substrate by the preferred component is not much affected by the variance in temperature as in the case $T=0.60,0.70$ and 0.80 . This applies for only partial wetting of the substrates. The higher the average composition of the two components, the higher is the value of the 
average number density perpendicular to the two walls (Fig. 7b).

\section{Conclusion}

This paper work has mainly focused to obtain the rhythmic and polynomial fitting periodic behavior as observed in the case of 2D conserved binary random mixture with 70:30 composition. However, the exact observation could not be obtained with single precession program because of the values obtained are at the 6-7th decimal places, if generalized formalism of 2D (Pearson's linear correlation function) is used in 3D. But the 3D simulation confirm the two regime behavior indicating that the same colossal behavior can exist in real $3 \mathrm{D}$ thin film of random binary mixture. Thus, the colossal behavior as obtained for the case of 2D problem is retained, and this may indicate for a quantized or discrete colossal behavior for certain set of composition and interface parameters.

Open Access This article is distributed under the terms of the Creative Commons Attribution License which permits any use, distribution and reproduction in any medium, provided the original author(s) and source are credited.

\section{References}

Binder K, Heermann DW (1998) Monte Carlo simulation in statistical physics, 2nd edn. Springer, Heidelberg, p 29, 99

Binder K, Nielaba P, Pereyra V (1997) Phase coexistence in binary mixtures in thin films with symmetric walls: model calculations for two- and three-dimensional Ising lattices. Z Phys B 104:81

Bischof J, Scherer D, Herminghaus S, Leiderer P (1996) Dewetting modes of thin metallic films: nucleation of holes and spinodal dewetting. Phys Rev Lett 77:1536-1539
Bonn D, Ross D (2001) Wetting transitions. Rep Prog Phys 64:1085

Christenson HK (2001) Confinement effects on freezing and melting. J Phys Condens Matter 13:R95

Goldenfeld N (1992) In: Lectures on phase transitions and the renormalization group, Frontiers in Physics, pp 86-88

Henderson JR (1999) Statistical mechanics of patterned in homogeneous fluid phenomena. J Phys Condens Matter 11:629

Herminghaus S et al (1998) Spinodal dewetting in liquid crystal and liquid metal films. Science 282:916-919

Press WH et al (1998) Numerical recipes, 2nd edn. Cambridge University Press, Cambridge

Puri S, Binder K (1992) Surface-directed spinodal decomposition: phenomenology and numerical results. Phys Rev A 46:R4487R4489

Reiter G (1992) Dewetting of thin polymer films. Phys Rev Lett 68:75-78

Reiter G (1998) Thin-film pattern formation: the artistic side of intermolecular forces. Science 282:888-889

Sharma A, Khanna R (1998) Pattern formation in unstable thin liquid films. Phys Rev Lett 81:3463-3466

Singh SP (2010) A review of molecular simulation: future scope and limitations in synthesis and characterization of nanostructured materials. In: Proceedings of the International Conference on Nanomaterials and Nanotechnology, at KSRCT, Erode Tamilnadu, India, Macmillan Advanced Research Series, 13-16 Dec 2010, p 417

Singh SP (2011) Spinodal theory: a common rupturing mechanism in spinodal dewetting and surface directed phase separation (some technological aspects and the significance of dipole-quadrupole interaction in spinodal dewetting). Adv Condens Matter Phys, vol 2011, Manuscript ID 526397

Singh SP, Singh K, Roychoudhury M (2008) Monte Carlo simulation for diffusion limited surface directed phase separation. In: Proceedings of National Academy of Sciences A, Part-I, vol 78, pp 79-83

Wilding NB (2003) Continuous demixing at liquid-vapor coexistence in a symmetrical binary fluid mixture. Phys Rev E 67:052503

Woywod D, Schoen M (2004) The wetting of planar solid surfaces by symmetric binary mixtures near bulk gas-liquid co-existence. J Phys Condens Matter 16:4761

Xie R, Karim A, Douglas JF, Han CC, Weiss RA (1998) Spinodal dewetting of thin polymer films. Phys Rev Lett 81:1251-1254 